12,01

\title{
Локализация возбуждений в слоистой структуре с границами раздела, характеризующимися нелинейным откликом
}

\author{
(C) C.E. Савотченко \\ Белгородский государственный технологический университет им. В.Г. Шухова, \\ Белгород, Россия \\ E-mail: savotchenkose@mail.ru
}

(Поступила в Редакцию 11 ноября 2018 г.)

Рассмотрены особенности локализации возбуждений в трехслойной структуре, в которой линейные среды разделены границами, характеризующимися собственным нелинейным откликом. Показано, что в рассматриваемой трехслойной структуре могут существовать локализованные состояния двух типов, различающиеся распределением поля во внутреннем слое, а также частотным диапазоном существования. Для каждого случая получены дисперсионные соотношения, определяющие зависимости энергии от параметров системы. Получены коэффициенты затухания поверхностных волн в явном виде. Указаны условия локализации поля в зависимости от характеристик слоев и границ их раздела. Найдены энергии локализованных состояний, которые не существуют в симметричной структуре без взаимодействия волны с границами раздела слоев, причем обязательным является наличие нелинейного отклика границ. Показано, что наличие взаимодействия волны с границами раздела слоев приводит к тому, что в одномерной симметричной потенциальной яме с бесконечно высокими стенками с нелинейным откликом локализованное состояние существует не всегда. Проанализировано влияние параметров сред и границ их раздела на поток, переносимый поверхностными волнами.

DOI: $10.21883 /$ FTT.2019.03.47253.288

\section{1. Введение}

Вопросам существования поверхностных волн и локализации возбуждений вблизи границ раздела сред, в том числе и нелинейных, посвящено большое количество литературы [1-3]. В последние годы возрос интерес к теоретическому исследованию эффектов, обусловленных существованием локализованных состояний в нелинейных средах вблизи разнообразных видов дефектов, характеризующихся различными физическими условиями и природой их формирования [4].

Для теоретического описания подобных явлений широко используются нелинейные дифференциальные уравнения, среди которых особое место занимает нелинейное уравнение Шредингера (НУШ) [5]. К уравнениям, имеющим форму подобную НУШ, сводятся многие модели физических систем различной природы $[6,7,8]$. К примеру, в [9] в рамках теории бозе-эйнштейновской конденсации описаны экситоны в кольцевых ловушках при помощи уравнения Гросс-Питаевского, формально эквивалентного НУШ [10].

Также можно отметить, что в рамках теории Гинзбурга-Ландау минимизация функционала свободной энергии в приближении среднего поля приводит нелинейному уравнению, совпадающему по своей структуре со стационарным НУШ, коэффициенты которого зависят от температуры и параметров зонной структуры [11-13]. В указанных работах дефекты, обуславливающие локализацию состояний, моделировались при помощи прямоугольных потенциальных ям или короткодействующего потенциала с дельта-функцией Дирака.
Также предлагались различные обобщения потенциалов, описывающих дефекты с дополнительными свойствами [14-16]. К примеру, в $[17,18]$ использовалось НУШ с нелинейностью только внутри узкого волновода для формулировки модели оптической системы, в которой периодическая модуляция линейного показателя преломления сочетается с одиночным нелинейным дефектом. Была рассмотрена модель нелинейной оптической среды с эффектом Керра, содержащей периодически расположенные дефектные слои, характеризующиеся показателем преломления, сильно отличающимся от показателя преломления оптической среды других слоев между ними. В [19] в рамках такой модели рассматривалась локализация возбуждений поля вблизи одиночного нелинейного дефекта, играющего роль нелинейного волновода в оптической линейной среде.

К уравнению, формально совпадающему со стационарным НУШ без потенциала, сводятся модели оптических сред как с керровской нелинейностью [20], так и с другими видами нелинейной зависимости диэлектрической функции от напряженности электрического поля [21-24]. Из многослойных сред наиболее часто рассматривается трехслойная структура, состоящая из сред с различными физическими характеристиками [25-27].

В настоящей работе будет рассматриваться модель трехслойной структуры, слои которой представляют собой линейные среды, а нелинейность будет учитываться только внутри ультратонких границ их раздела [19]. Основной целью данной работы является описание на основе НУШ новых особенностей локализации поверхностных волн вблизи нелинейных плоских дефектов, 
в качестве которых выступают ультратонкие границы раздела линейных сред, которые сами характеризуются нелинейными свойствами.

Для описания новых особенностей взаимодействия волн и границ с учетом их нелинейных свойств в данной работе предлагается использовать нелинейный самосогласованный потенциал, который зависит от квадрата амплитуды искомого поля, и для случая одиночной границы использовался в работах [28-36]. При наличии слабой связи между плоскопараллельными волноводами, амплитуды поля в которых существенно превышает усредненное значение амплитуды поля во всем кристалле, нелинейные слагаемые в НУШ могут учитываться только в внутри самих волноводов [17-19].

Следует подчеркнуть, что в [19] рассматривалась одиночная граница с нелинейными свойствами, а в данной работе - две плоскопараллельные границы, что будет привносить новые особенности локализации состояний, связанные с взаимным влиянием таких факторов, как расстояние между границами и интенсивности их нелинейного отклика.

Будут описаны локализованные состояния, возникающие в двух различных диапазонах в зависимости от различия характеристик внутреннего и внешних слоев. В явном аналитическом виде будут получены зависимости коэффициентов затухания волн, энергии, амплитуд и потоков от характеристик сред, интенсивности взаимодействия волны с границами раздела и их нелинейным откликом.

\section{2. Уравнения модели}

Рассмотрим трехслойную структуру, в которой внутренний слой толщины $2 a$ разделяет два кристалла. Границы раздела сред считаются плоскими и много меньше характерного масштаба локализации возмущений параметров среды, создаваемых ими. Ширина внутреннего слоя считается существенно больше ширины одной границы раздела слоев.

Систему координат выберем так, чтобы средина внутреннего слоя проходила через начало координат. Границы раздела слоев лежали в плоскостях $x= \pm a$ перпендикулярно оси $x$.

Для описания интересующих нас свойств и условий их проявления используем одномерное стационарное НУШ, которому подчиняется поле $\psi$ с энергией стационарного состояния $E$

$$
E \psi=-\psi_{x x}^{\prime \prime} / 2 m+\Omega(x) \psi+U\left(x,|\psi|^{2}\right) \psi .
$$

Характеристики слоев $\Omega(x)$ будем аппроксимировать кусочно-постоянной функцией: $\Omega(x)=\Omega_{1}, x<-a$, $\Omega(x)=\Omega_{0},|x|<a, \Omega(x)=\Omega_{2}, x>a, \Omega$ - постоянные величины, $j=0,1,2$.

Будем считать, что среда внутри самой границы раздела характеризуется своим откликом (взаимодействием с возбуждением), который и определяют фор- му потенциала $U$. Тогда в силу того, что толщина границы существенно меньше характерной длины локализации возмущений поля, локальный потенциал в короткодействующем („,точечном“) приближении можно аппроксимировать дельта-функцией Дирака $\delta(x)$. В данной работе, как и в [19], будем также считать, что нелинейной зависимостью от амплитуды поля обладает среда только внутри границ раздела слоев. Поэтому потенциал, описывающий нелинейные свойства границ раздела, будем рассматривать в виде [36]

$$
\begin{array}{r}
U\left(x,|\psi|^{2}\right)=F_{1}\left(x+a,|\psi|^{2}\right)+F_{2}\left(x-a,|\psi|^{2}\right), \\
F_{j}\left(x,|\psi|^{2}\right)=\left\{U_{j}+W_{j}|\psi|^{2}\right\} \delta(x), \quad j=1,2,
\end{array}
$$

$U_{j}$ - интенсивности взаимодействия возбуждений с границами раздела в линейном приближении („мощности“ дефектов). При $U_{j}>0$ возбуждения отталкиваются от соответствующей границы, а при $U_{j}<0-$ притягиваются. Параметры нелинейности границ раздела слоев $W_{j}$ характеризуют нелинейный отклик их взаимодействия с возбуждениями. При $W_{j}>0$ соответствующая граница обладает внутренней дефокусировкой, а при $W_{j}<0$ - самофокусировкой. В общем случае в силу различия параметров сред всех слоев все эти величины будем считать различными.

Нахождение решения НУШ (1) с потенциалом (2) эквивалентно решению УШ без потенциала

$$
\psi_{x x}^{\prime \prime}+2 m(E-\Omega(x)) \psi=0,
$$

с граничными условиями

$$
\begin{gathered}
\psi( \pm a-0)=\psi( \pm a+0)=\psi_{0 j}, \\
\psi^{\prime}( \pm a+0)-\psi^{\prime}( \pm a-0)=2 m\left\{U_{j}+W_{j}\left|\psi_{0 j}\right|^{2}\right\} \psi_{0},
\end{gathered}
$$

где $\psi_{0 j}=\psi( \pm a)$ - амплитуды колебаний поля на границах раздела слоев.

Здесь и далее значение индекса $j=1$ соответствует величинам, относящимся к области $x<-a, j=2-$ к области $x>a, j=0-$ к внутреннему слою при $|x|<a$. При этом в формуле (5) и далее для $j=1$ следует выбирать нижний знак, а для $j=2-$ верхний.

Таким образом, математическая формулировка модели сводится к контактной краевой задаче для уравнения (3) с граничными условиями (4) и (5).

В случае одной границы раздела в плоскости $x=0$ при $U_{j}=0, W_{j}=W_{0}$ и $a=0$ для случая нелинейного плоского дефекта из (5) получается одно граничное условие, приведенное в [28-30], а для случая обоих ненулевых параметров $\left(U_{j}=U_{0}, W_{j}=W_{0}\right)$ - в [3133]. Для системы двух плоскопараллельных дефектов с линейным взаимодействием при $U_{j}=U_{0}, W_{j}=0$ и $a \neq 0$ из (5) получаются граничные условия, приведенные в [7]. При $U_{j}=0, W_{j} \neq 0$ и $a \neq 0$ из (5) получаются граничные условия, использованные в [36] для двух плоскопараллельных дефектов с преобладающим нелинейным откликом. В этой работе был проанализирован 
только случай одинаковых значений соответствующих характеристик слоев и их границ раздела, в отличие от приведенных здесь результатов.

В данной работе будут рассматриваться только локализованные в пространстве решения сформулированной краевой задачи (3)-(5), которые удовлетворяют условию исчезновения на бесконечности $|\psi(x)| \rightarrow 0$ при $x \rightarrow \infty$. Такие решения описывают распределение поля в бегущей вдоль границ раздела волне, и быстро затухающее при удалении от них.

Физическая применимость моделей, использующих НУШ (1) с нелинейным самосогласованным потенциалом, неоднократно обсуждалась ранее в работах [7,32,33,35]. К примеру, в [12,13] НУШ с кубической нелинейностью применялось для описания структуры ближнего антиферромагнитного порядка, индуцированного перераспределением зарядовой плотности вблизи границы радела слоев $\mathrm{Fe} / \mathrm{Cr}$ в композитной многослойной структуре. В данной интерпретации волновая функция $\psi$ представляет собой параметр антиферромагнитного порядка, равный произведению амплитуды линейно поляризованной волны спиновой плотности на ее эффективный потенциал, зависящий от выбора микроскопической модели. Обобщение данной модели на случай дефекта с нелинейным откликом было предложено в [34] для контакта нелинейных сред, а в [35] для контакта линейной и нелинейной сред.

Другим физическим примером использования рассматриваемой модели может служить контакт оптических немагнитных сред с керровской нелинейностью, возмущение электрического поля в которых описывается НУШ с короткодействующим линейным потенциалом [7]. Обобщение такой модели для случая, когда узкие дефектные слои характеризуются нелинейным показателем преломления, сильно отличающимся от показателя преломления оптической среды широких слоев, предложено в [32].

Как отмечалось выше, НУШ в форме (1) наиболее часто применяется в нелинейной оптике для описания различных типов волн, в том числе и локализованных вблизи границ раздела сред, представляющих собой тонкопленочные слои с отличающимися коэффициентами преломления и играющие роль волноводов. К примеру, в $[17,18]$ рассматривались ТЕ-поляризованные монохроматические электромагнитные волны, распространяющиеся вдоль границы раздела (плоскости $y z$ ). Считалось, что можно разделить переменные и представить $y$-вую компоненту напряженности электрического поля в виде $E_{y}=E_{y}(x, z) E_{y}(y)$. Тогда, как показано в [18], распределение поля $E_{y}(x, z)$ будет описываться НУШ

$$
i \frac{\partial E_{y}}{\partial z}+D_{e} \frac{\partial^{2} E_{y}}{\partial x^{2}}+n_{L}(x) E_{y}+n_{N}\left(x,\left|E_{y}\right|^{2}\right) E_{y}=0,
$$

где $D_{e}$ - коэффициент дифракции, $n_{L}$ и $n_{N}$ - линейный и нелинейный показатели преломления соответственно. НУШ (6) описывает распределение светового пучка в рамках так называемого параболического приближения, справедливого для волн, распространяющихся вдоль направления $z$. Данное приближение соответствует тому, что характерная длина искажения пучка, обусловленная как дифракцией, так и преломлением вдоль оси $z$, должна быть намного больше ширины пучка в поперечном направлении $x$. Индуцированные нелинейностью в профиле электрического поля $E_{y}(y)$ изменения должны быть пренебрежительно малыми. Такие условия могут реализовываться в оптических средах с низким коэффициентом поглощения, примером которых могут быть плоскопараллельные волноводные структуры AlGaAs [37].

Здесь предполагается, что основная среда является оптически линейной, а нелинейные свойства проявляются только внутри тонкопленочного волновода. Если соответствующая ширина внутреннего слоя структуры намного больше, чем ширина волновода, то по аналогии с приближением Кронига-Пенни в теории твердого тела, тонкопленочный волновод может быть смоделирован дельта-функцией, который в простейшем случае может описывать одиночный нелинейный граничный слой. Данное приближение оправдано в том случае, когда характерный масштаб локализации возмущений параметров среды, создаваемых границей раздела, существенно превосходит толщину тонкопленочного волновода. В результате можно было записать потенциал в форме (2). Модель, использующая дельта-функцию, позволяет выявить аналитически характерные особенности таких нелинейных систем. Кроме того, такую модель можно будет легко обобщить на случай периодического массива тонкопленочных нелинейных волноводов.

Из НУШ (6) вытекает физический смысл коэффициентов модельного НУШ (1) для случая оптической системы. В частности, „эффективная масса“ возбуждения определяется коэффициентом дифракции по формуле $m=1 / 2 D_{e}$, энергия $E$ в (1) имеет смысл константы распространения, функция $\Omega(x)$ прямо пропорциональна линейному показателю преломления в широких слоях, а параметры $U_{j}-$ в тонкопленочных волноводах, параметры $W_{j}$ пропорциональны нелинейным показателям преломления (коэффициентам керровской нелинейности) в тонкопленочных волноводах, характеризующие их нелинейный отклик [32].

\section{3. Поверхностные волны в системе плоскопараллельных нелинейных волноводов}

\section{1. Поверхностные волны первого типа}

Если значение энергии лежит в диапазоне $E<\min \left\{\Omega_{1}, \Omega_{2}, \Omega_{0}\right\}$, то решение краевой задачи $(3)-(5)$

$$
\begin{array}{cc}
\psi(x)=\psi_{0 j} \exp \left(\mp q_{j}(x \mp a)\right), & |x|>a \\
\psi(x)=A \operatorname{ch}\left(q_{0} x\right)+B \operatorname{sh}\left(q_{0} x\right), & |x|<a,
\end{array}
$$


где коэффициенты затухания

$$
q_{j}^{2}=2 m\left(\Omega_{j}-E\right) .
$$

Из граничных условий (4) и (5) следует связь амплитуд

$$
\begin{gathered}
\psi_{0 j}=A \operatorname{ch}\left(q_{0} a\right) \pm B \operatorname{sh}\left(q_{0} a\right), \\
q_{0}\left\{A \operatorname{sh}\left(q_{0} a\right) \pm B \operatorname{ch}\left(q_{0} a\right)\right\}+\psi_{0 j}\left(q_{j}+d_{j}\right)=0,
\end{gathered}
$$

где обозначили $d_{j}=2 m\left(U_{j}+W_{j}\left|\psi_{0 j}\right|^{2}\right)$.

В трехслойной структуре существуют синфазные и противофазные колебательные состоянии. Для синфазных колебаний амплитуды в обеих плоскостях на границах раздела слоев одинаковы

$$
\psi_{01}=\psi_{02}=A \operatorname{ch}\left(q_{0} a\right)=\psi_{0} \quad \text { и } \quad B=0 .
$$

Для противофазных колебаний амплитуды в обеих плоскостях на границах раздела слоев одинаковы по абсолютной величине, но противоположны по знаку

$$
\psi_{01}=-\psi_{02}=-B \operatorname{sh}\left(q_{0} a\right)=\psi_{0} \quad \text { и } \quad A=0 .
$$

\section{2. Поверхностные волны второго типа}

Если значение энергии лежит в диапазоне $\Omega_{0}<E<\min \left\{\Omega_{1}, \Omega_{2}\right\}$, то решение краевой задачи (3)-(5) описывается функциями (7) во внешних полупространствах, а во внутреннем слое - выражением

$$
\psi(x)=A \cos (k x)+B \sin (k x), \quad|x|<a,
$$

где волновое число

$$
k^{2}=2 m\left(E-\Omega_{0}\right)
$$

Из граничных условий (4) и (5) следует связь амплитуд

$$
\begin{gathered}
\psi_{0 j}=A \cos (k a) \pm B \sin (k a), \\
k\{A \sin (k a) \pm B \cos (k a)\}=\psi_{0 j}\left(q_{j}+d_{j}\right) .
\end{gathered}
$$

Также как и в случае локализованных состояний первого типа, описываемых во внутреннем слое функцией (8), существуют синфазные и противофазные колебательные состояния второго типа. Для синфазных колебаний амплитуды в обеих плоскостях на границах раздела слоев одинаковы

$$
\psi_{01}=\psi_{02}=A \cos (k a)=\psi_{0} \quad \text { и } \quad B=0 .
$$

Для противофазных колебаний

$$
\psi_{01}=-\psi_{02}=-B \sin (k a)=\psi_{0} \quad \text { и } \quad A=0 .
$$

\section{4. Энергии и условия локализации состояний в абсолютно симметричной трехслойной структуре}

\section{1. Локализация волн первого типа}

Абсолютно симметричной будем называть такую трехслойную структуру, в которой внешние слои характеризуются одинаковыми значениями $\Omega_{1}=\Omega_{2}=\Omega$, а границы раздела - одинаковыми значениями своих параметров $U_{1}=U_{2}=U_{0}$ и $W_{1}=W_{2}=W_{0}$ соответственно. Коэффициенты затухания поверхностной волны в левом и правом полупространствах, как следует из (9), одинаковы: $q_{1}=q_{2}=q$.

Сначала рассмотрим синфазные состояния, определяемые условиями (12). В симметричной структуре из (10) и (11) получается дисперсионное уравнение

$$
q_{0} \operatorname{th}\left(q_{0} a\right)+q+d_{0}=0
$$

определяющее энергию локализации как функцию параметров системы, здесь обозначено $d_{0}=2 m\left(U_{0}+W_{0}\left|\psi_{0}\right|^{2}\right)$.

Можно отметить следующую особенность локализованных состояний с энергией, определяемой (20). Если оба параметра границ раздела равны нулю $\left(U_{0}=0\right.$ и $\left.W_{0}=0\right)$, но характеристики внешних и внутреннего слоев различны $\left(\Omega_{0} \neq \Omega\right)$, то дисперсионное соотношение (20) принимает вид $q_{0} \operatorname{th}\left(q_{0} a\right)+q=0$. Такая ситуация соответствует хорошо изученному случаю, когда нормальные производные поля непрерывны при переходе через границы раздела слоев [25]. Точно такое же дисперсионное уравнение получается, если оба параметра границ раздела не равны нулю $\left(U_{0} \neq 0\right.$ и $\left.W_{0} \neq 0\right)$, но амплитуда поля на границах раздела будет равна

$$
\left|\psi_{0}\right|^{2}=-U_{0} / W_{0}
$$

Энергии таких локальных состояний, очевидно, одинаковы, но существуют они в принципиально различных случаях. Во втором случае граница раздела обязательно должна обладать дополнительным нелинейным откликом, характеризуемым параметром $W_{0}$. Знаки параметров границы раздела слоев должны быть противоположным. Это означает, что локализация такого состояния с амплитудой (21) возможна вблизи притягивающей границы с дефокусирующим нелинейным откликом или вблизи отталкивающей границы с самофокусирующим нелинейным откликом.

В длинноволновом приближении при $q_{0} a \ll 1$ из дисперсионного уравнения (20) можно получить коэффициент затухания в виде

$$
q=\left\{\left[1+8 m a\left\{a\left(\Omega-\Omega_{0}\right)-U_{0}-W_{0}\left|\psi_{0}\right|^{2}\right\}\right]^{1 / 2}-1\right\} / 2 a .
$$

Требование вещественности и положительности коэффициента затухания определяет условие локализации 
длинноволновых синфазных состояний в абсолютно симметричной трехслойной структуре: $\left|\psi_{0}\right|^{2}<\left\{a\left(\Omega-\Omega_{0}\right)\right.$ $\left.-U_{0}\right\} / W_{0}$.

При $U_{0}=0$ и $W_{0}=0$, но $\Omega_{0} \neq \Omega$, из (22) следует, что локализация возможна при $\Omega>\Omega_{0}$, что соответствует прямоугольной потенциальной яме конечной ширины.

Если параметры всех слоев одинаковы, то есть $\Omega_{0}=\Omega$, и отсутствует нелинейный отклик в границе $\left(U_{0} \neq 0\right.$ и $\left.W_{0}=0\right)$, то из (22) следует, что локализация возможна только вблизи притягивающей границы, как обычно и получается в случае бесконечно глубокой потенциальной ямы.

Если граница характеризуется ярко выраженным нелинейным откликом $\left(\Omega_{0}=\Omega, U_{0}=0\right.$ и $\left.W_{0} \neq 0\right)$, то из (22) следует, что локализация возможна только вблизи границы с самофокусировкой.

Если оба параметра границ раздела не равны нулю $\left(\Omega_{0}=\Omega, U_{0} \neq 0\right.$ и $\left.W_{0} \neq 0\right)$, то из (22) следует, что локализация возможна, не только при противоположных знаках параметров границ, как отмечалось выше для колебаний с амплитудой (21), но и когда оба параметра отрицательны, то есть вблизи отталкивающей границы с дефокусирующим нелинейным откликом.

В противоположном предельном случае $q_{0} a \gg 1$ из дисперсионного уравнения (20) при $\Omega_{0}=\Omega$ получается коэффициент затухания $q=-d_{0} / 2$.

Теперь рассмотрим противофазные колебательные состояния, определяемые условиями (13). В симметричной структуре из (10) и (11) в этом случае получается дисперсионное уравнение

$$
q_{0} \operatorname{cth}\left(q_{0} a\right)+q+d_{0}=0 .
$$

Также как и для синфазных колебательных состояний, из (23) получается одно и тоже дисперсионное уравнение $q_{0} \operatorname{cth}\left(q_{0} a\right)+q=0$ в двух различных случаях:

1) параметры границ раздела не равны нулю $\left(U_{0} \neq 0\right.$ и $\left.W_{0} \neq 0\right)$, и амплитуда поля на границах раздела определяется выражением (21);

2) оба параметра границ раздела равны нулю $\left(U_{0}=0\right.$ и $\left.W_{0}=0\right)$, но характеристики внешних и внутреннего слоев различны $\left(\Omega_{0} \neq \Omega\right)$.

В длинноволновом приближении при $q_{0} a \ll 1$ из дисперсионного уравнения (23) можно получить коэффициент затухания в виде

$$
q=-\left\{1 / a+2 m\left(U_{0}+W_{0}\left|\psi_{0}\right|^{2}\right)\right\} .
$$

Локализация длинноволновых противофазных состояний в абсолютно симметричной трехслойной структуре происходит при условии: $\left|\psi_{0}\right|^{2}<-\left(U_{0}+1 / 2 m a\right) / W_{0}$. Данное условие выполняется в двух случаях

1) если граница характеризуется самофокусирующим нелинейным откликом, то граница может быть как притягивающей, так и отталкивающей, но должно выполняться условие $U_{0}>-1 / 2 m a$;
2) если граница характеризуется дефокусирующим нелинейным откликом, то граница может быть только притягивающей, причем $U_{0}<-1 / 2 m a$.

Если отсутствует нелинейный отклик в границе $\left(U_{0} \neq 0\right.$ и $\left.W_{0}=0\right)$, то из (24) следует, что $q=-\left(1 / a+2 m U_{0}\right)$, а значит, локализация возможна только вблизи притягивающей границы при $U_{0}<-1 / 2 m a$, как обычно и получается в случае бесконечно глубокой потенциальной ямы.

В противоположном предельном случае $q_{0} a \gg 1$ из дисперсионного уравнения (23) получается такой же уровень энергии, как и для синфазных колебаний, что естественно, так как на больших расстояниях от границ раздела (при „высоких“ энергиях) тип фазы колебательных состояний роли не играет.

\section{2. Локализация волн второго типа}

Сначала рассмотрим синфазные состояния второго типа, определяемые условиями (18). В симметричной структуре из (16) и (17) получается дисперсионное уравнение

$$
k \operatorname{tg}(k a)=q+d_{0},
$$

определяющее энергию локализации как функцию параметров системы.

Также как и для состояний первого типа, можно отметить следующую особенность локализованных состояний с энергией, определяемой (25). При $U_{0}=0$ и $W_{0}=0$ дисперсионное соотношение (25) принимает вид $k \operatorname{tg}(k a)=q$, которое с другой стороны, также можно получить из (25) при $U_{0} \neq 0$ и $W_{0} \neq 0$, но амплитуда поля на границах раздела будет фиксирована параметрами границ раздела и определяться выражением (21).

В длинноволновом приближении при $k a \ll 1$ из дисперсионного уравнения (25) можно получить коэффициент затухания в виде (22). Волновое число в этом случае будет связано с коэффициентом затухания (22) соотношением: $k^{2}=\left(q+d_{0}\right) / a$. Если энергия $E$ близка еще и к уровню $\Omega$ (что возможно в узкой полосе при малом различии характеристик внутреннего и внешних слоев), а интенсивность взаимодействия с границей высока, то из этого выражения получается волновое число $k^{2}=d_{0} / a$, соответствующее энергии $E=\Omega_{0}+d_{0} / 2 m a$. Для существования такого состояния оба параметра границы должны быть положительными, то есть граница должна характеризоваться отталкиванием и дефокусирующим нелинейным откликом.

Теперь рассмотрим противофазные колебательные состояния, определяемые условиями (19). В симметричной структуре из (16) и (17) в этом случае получается дисперсионное уравнение

$$
k \operatorname{ctg}(k a)+q+d_{0}=0 .
$$

Также как и для синфазных колебательных состояний, из (26) получается одно и то же дисперсионное 
уравнение $k \operatorname{ctg}(k a)+q=0$ в двух различных случаях, описанных выше.

В длинноволновом приближении при $k a \ll 1$ из дисперсионного уравнения (26) можно получить коэффициент затухания в виде (24).

Следует отметить, что уравнения (25) и (26) при $U_{0}=0$ и $W_{0}=0$ хорошо изучены, и известно, что существует не менее одного дискретного уровня энергии. Если же $U_{0} \neq 0$ и $W_{0} \neq 0$, что соответствует наличию взаимодействия волны с границей раздела, то условие существования локализованных синфазных и противофазных состояний меняются, и как отмечено в [9], в одномерной яме уже не всегда существует связанное состояние.

\section{5. Энергии и условия локализации состояний в несимметричной трехслойной структуре}

\section{1. Локализация волн первого типа с синфазными и противофазными колебаниями границ раздела слоев}

Несимметричной будем называть такую трехслойную структуру, в которой границы раздела характеризуются различными значениями своих соответствующих параметров $U_{1} \neq U_{2}$ и $W_{1} \neq W_{2}$, а внешние и внутренний слои при этом могут характеризоваться как одинаковыми значениями своих параметров $\Omega_{j}$, так и различными.

5.1.1. Синфазные состояния. В несимметричной структуре из (10) и (11) с учетом условий (12) получается система: $q_{0} \operatorname{th}\left(q_{0} a\right)+q_{j}+d_{0 j}=0$, $d_{0 j}=2 m\left(U_{j}+W_{j}\left|\psi_{0}\right|^{2}\right)$, определяющая энергию локализации как функцию параметров системы. Если характеристики внешних слоев совпадают $\left(\Omega_{1}=\Omega_{2}=\Omega\right)$, то из нее находится амплитуда

$$
\left|\psi_{0}\right|^{2}=\left(U_{2}-U_{1}\right) /\left(W_{1}-W_{2}\right)
$$

Как вытекает из (27), локализация возможна при выполнении одной из пар условий, определяющих соотношения между характеристиками двух границ раздела слоев: 1) $W_{1}>W_{2}$ и $U_{1}<U_{2}$ или 2) $W_{1}<W_{2}$ и $U_{1}>U_{2}$.

Следует подчеркнуть, что локализованные состояния с амплитудой синфазных колебаний поля на границах, определяемой (27), не существуют без взаимодействия волны с границами раздела слоев, причем обязательно наличие нелинейного отклика границ.

В длинноволновом приближении при $q_{0} a \ll 1$ получается коэффициент затухания синфазных состояний в несимметричной структуре в виде

$$
q=\left\{\left[1+8 m a\left(a\left(\Omega-\Omega_{0}\right)-D\right)\right]^{1 / 2}-1\right\} / 2 a,
$$

где $D=\left(W_{1} U_{2}-W_{2} U_{1}\right) /\left(W_{1}-W_{2}\right)$. Из (28) получается условие локализации длинноволновых синфазных состояний в несимметричной трехслойной структуре: $\Omega>\Omega_{0}+D / a$.
В противоположном предельном случае $q_{0} a \gg 1$ получается коэффициент затухания синфазных колебательных состояний в несимметричной структуре

$$
q=\left(\Omega_{0}-\Omega\right) / 2 D-m D
$$

При $\Omega_{0}=\Omega$ (29) упрощается: $q=-m D$. Следовательно, для локализации в этом случае $D<0$, что обеспечивается выполнением одной из пар условий, определяющих соотношения между характеристиками двух границ раздела слоев: 1) $1<W_{2} / W_{1}<U_{2} / U_{1}$ или 2) $U_{2} / U_{1}<W_{2} / W_{1}<1$.

При $\Omega_{0} \neq \Omega$ если эти условия не выполняются и будет $D>0$, тогда локализация возможна при $\Omega>\Omega_{0}+2 m D^{2}$. Если $D<0$, то для локализации достаточно $\Omega_{0}>\Omega$.

При различных характеристиках внешних слоев (когда $\left.\Omega_{1} \neq \Omega_{2}\right)$ получается энергия локализованных синфазных состояний в виде

$$
E=\left\{\Omega_{1} \Omega_{2}-\left(\Omega_{1}+\Omega_{2}-2 m \Delta^{2}\right)^{2} / 4\right\} / 2 m \Delta^{2},
$$

где $\Delta=U_{2}-U_{1}+\left(W_{2}-W_{1}\right)\left|\psi_{0}\right|^{2}$.

В пределе $|\xi| \ll 1$, где $\xi=\left(W_{2}-W_{1}\right) /\left(U_{2}-U_{1}\right)$, из (30) получается

$$
E=E_{l}-\alpha \xi\left|\psi_{0}\right|^{2}
$$

где $E_{l}=\alpha-2\left(\Omega_{1}+\Omega_{2}\right), \alpha=\left\{\Omega_{1} \Omega_{2}-\left(\Omega_{1}+\Omega_{2}\right)^{2} / 4\right\} /$ $2\left(U_{2}-U_{1}\right)$. Амплитуда таких колебательных состояний в основном порядке малости по $\xi$ определяется выражением

$$
\left|\psi_{0}\right|^{2}=-\left\{U_{1}+\left(q_{0 l} \operatorname{th}\left(q_{0 l} a\right)+q_{1 l}\right) 2 m\right\} / W_{1},
$$

где $q_{j l}^{2}=2 m\left(\Omega_{j}-E_{l}\right), j=0,1$. Энергия (31) зависит от квадрата амплитуды поля, как это обычно и бывает для нелинейных колебаний [2]. При отсутствии нелинейного отклика границ $\xi=0$ и состояния из нелинейных становятся линейными с $E=E_{l}$.

Таким образом, получается, что нелинейный отклик границ раздела слоев приводит к тому, что линейные волны обладают характерными для нелинейных волн свойствами, а именно - зависимостью энергии от квадрата амплитуды волны.

5.1.2. Противофазные состояния. В несимметричной структуре из (10) и (11) с учетом (13) в этом случае получается система: $q_{0} \operatorname{cth}\left(q_{0} a\right)+q_{j}+d_{0 j}=0$. При различных характеристиках внешних слоев $\left(\Omega_{1} \neq \Omega_{2}\right)$ из него можно получить энергию локальных состояний в форме, совпадающей с (30) и, соответственно, в пределе $|\xi| \ll 1$ в форме, совпадающей с (31). Отличие противофазных от синфазных колебательных состояний заключается в амплитуде, которая в основном порядке малости по $\xi$ имеет вид

$$
\left|\psi_{0}\right|^{2}=-\left\{U_{1}+\left(q_{0 l} \operatorname{cth}\left(q_{0 l} a\right)+q_{1 l}\right) / 2 m\right\} / W_{1} .
$$


Если характеристики внешних слоев совпадают $\left(\Omega_{1}=\Omega_{2}=\Omega\right)$, то в длинноволновом приближении при $q_{0} a \ll 1$ получается коэффициент затухания противофазных колебательных состояний в несимметричной структуре в виде

$$
q=-(1 / a+2 m D)
$$

Условие локализации длинноволновых противофазных колебательных состояний в несимметричной трехслойной структуре будет иметь вид: $W_{2}-W_{1}<$ $<2 m a\left(W_{1} U_{2}-U_{1} W_{2}\right)$. Выполнение такого условия можно добиться как при одинаковых, так и при различных знаках параметров границ раздела слоев. Видно, что для существования локальных длинноволновых противофазных колебательных состояний в несимметричной трехслойной структуре необходимо взаимодействие волны с границами раздела слоев (должно быть $D \neq 0$ ).

В противоположном предельном случае $q_{0} a \gg 1$ для противофазных колебательных состояний получается такой же коэффициент затухания, как и для синфазных состояний (29), что и должно быть в данном переделе, поскольку на больших расстояниях вдали от границы спектра энергии таких состояния совпадают.

\section{2. Локализация волн второго типа}

Сначала рассмотрим синфазные состояния. В несимметричной структуре получается система дисперсионных уравнений: $k \operatorname{tg}(k a)=q_{j}+d_{0 j}$. Если характеристики внешних слоев совпадают $\left(\Omega_{1}=\Omega_{2}=\Omega\right)$, то амплитуда будет определяться выражением (27). Условия локализации в этом случае остаются такими же, как и в п. 5.1.1.

В длинноволновом приближении при $k a \ll 1$ можно получить волновое число в случае, если энергия $E$ близка еще и к уровню $\Omega$, а интенсивность взаимодействия с границей велика: $k^{2}=2 m D / a$. Тогда соответствующее значение энергии будет $E=\Omega_{0}+D / a$. Для существования такого состояния должно быть $D>0$, что обеспечивается выполнением одной из пар условий, определяющих соотношения между характеристиками двух границ раздела слоев: 1) $W_{2}<W_{1}$ и $W_{1} / W_{2}>U_{1} / U_{2}$ или 2) $W_{2}>W_{1}$ и $W_{1} / W_{2}<U_{1} / U_{2}$.

Теперь рассмотрим противофазные колебательные состояния, определяемые (13). В несимметричной структуре из (10) и (11) с учетом (13) в этом случае получается система: $k \operatorname{ctg}(k a)+q_{j}+d_{0 j}=0$. Если $\Omega_{1}=\Omega_{2}=\Omega$, то амплитуда также будет определяться выражением (27).

Приведенные здесь дисперсионные уравнения, определяющие синфазные и противофазные состояния в несимметричной структуре, отличаются от дисперсионных уравнений, определяющие синфазные и противофазные состояния в симметричной структуре, тем, что энергии состояний в несимметричной структуре выражаются через параметры системы и не зависят от амплитуды поля (которая, напомним, выражена в виде (27) через параметры границ раздела слоев), а энергии состояний в симметричной структуре зависят от квадрата амплитуды поля на границах.

\section{6. Потоки энергии, переносимые поверхностными волнами}

\section{1. Полный поток}

Несмотря на то, что поверхностные волны в трехслойных структурах рассматривались неоднократно [7,21,25-27], остается не изученным взаимное влияние на переносимый поверхностными волнами вдоль слоев поток различия характеристик границ раздела и интенсивности взаимодействия волн с ними в многослойных структурах.

Рассмотрим сохраняющийся вдоль слоев поток энергии, переносимый поверхностной волной, который представляет собой первый интеграл НУШ (1):

$$
N=\int_{-\infty}^{+\infty}|\psi(x)|^{2} d x
$$

Выражение (35) может трактоваться с точки зрения теории бозе-эйнштейновской конденсации как полное число частиц в системе $[9,10]$. Кроме того, можно рассматривать (35) как условие нормировки, считая поток фиксированным. Тогда поток может выступать в роли управляющего параметра, через который можно выразить какие-либо другие характеристики локализованных состояний.

Вычисление потока энергии поверхностных волн по формуле (35) приводит к выражению, которое можно представить в виде суммы трех частей: $N=N_{0}+N_{1}+N_{2}$, где $N_{0}$ - поток во внутреннем слое при $|x|<a, N_{1}$ - поток в левом внешнем полупространстве при $x<-a, N_{2}$ - поток в правом внешнем полупространстве при $x>a$.

Поскольку в данной работе основное внимание уделяется анализу влияния взаимодействия волн с нелинейными границами раздела слоев, то далее ограничимся рассмотрением случая, когда параметры сред во внешних полупространствах несимметричной трехслойной структуры одинаковы $\left(\Omega_{1}=\Omega_{2}=\Omega\right)$, а параметры двух ее границ различны $\left(U_{1} \neq U_{2}\right.$ и $\left.W_{1} \neq W_{2}\right)$.

\section{2. Компоненты потоков во внешних полупространствах}

Потоки во внешних полупространствах для волн первого и второго типов представимы в виде

$$
N_{j}=\left|\psi_{0 j}\right|^{2} / 2 q_{j}, \quad j=1,2 .
$$

Поскольку по определению коэффициенты затухания во внешних слоях положительны, то форма потоков (36) показывает, что они также будут положительными. 
Для длинноволновых синфазных колебательных состояний, подставив амплитуду (27) и коэффициент затухания (28) в (36), можно получить поток в виде

$$
N_{j}=\frac{a\left(U_{2}-U_{1}\right)}{\left\{\left[1+8 m a\left(a\left(\Omega-\Omega_{0}\right)-D\right)\right]^{1 / 2}-1\right\}\left(W_{1}-W_{2}\right)},
$$

который в обоих внешних полупространствах стал одинаков.

Если характеристики сред во внутреннем и внешних слоях одинаковы (то есть $\Omega_{0}=\Omega$ ), то (37) примет вид

$$
N_{j}=\frac{a\left(U_{2}-U_{1}\right)}{\left\{[1-8 m a D]^{1 / 2}-1\right\}\left(W_{1}-W_{2}\right)} .
$$

В пределе $m a D \ll 1$ из (38) получается оценка потока

$$
N_{j}=\frac{\left(U_{2}-U_{1}\right)}{4 m\left(U_{1} W_{2}-U_{2} W_{1}\right)} .
$$

Для синфазных колебательных состояний в пределе $q_{0} a \gg 1$, подставив амплитуду (27) и коэффициент затухания (29) в (36), можно получить поток в виде

$$
N_{j}=\frac{a\left(U_{2}-U_{1}\right) D}{\left(W_{1}-W_{2}\right)\left(\Omega_{0}-\Omega-2 m D^{2}\right)} .
$$

При выполнении условий локализации состояний, полученных в п. 5.1.1, оценки потоков (38)-(40) будут положительными.

Если характеристики сред во внутреннем и внешних слоях одинаковы, то (40) примет вид

$$
N_{j}=\frac{\left(U_{2}-U_{1}\right)}{2 m\left(U_{1} W_{2}-U_{2} W_{1}\right)} .
$$

Можно отметить, что поток (41), полученный в пределе $q_{0} a \gg 1$, отличается в два раза от потока (39), полученного в длинноволновом пределе $q_{0} a \ll 1$ при дополнительном требовании малости $m a D \ll 1$.

Для длинноволновых противофазных колебательных состояний, подставив амплитуду (27) и коэффициент затухания (32) в (36), можно получить поток

$$
N_{j}=\frac{a\left(U_{1}-U_{2}\right)}{2\left(W_{1}-W_{2}+2 m a\left(U_{1} W_{2}-U_{2} W_{1}\right)\right)} .
$$

При выполнении условий локализации состояний, полученных в п. 5.1.2, поток (42) будет положительным.

В пределе $m a D \ll 1$ из (42) получается оценка потока

$$
N_{j}=\frac{a\left(U_{1}-U_{2}\right)}{2\left(W_{1}-W_{2}\right)} .
$$

Учитывая (27) и (43), можно указать оценку данного потока в виде $N_{j}=a\left|\psi_{0}\right|^{2} / 2$.

Для противофазных колебательных состояний в пределе $q_{0} a \gg 1$ получается такая же оценка потока во внешних слоях, как и для синфазных в виде (40).

\section{3. Поток во внутреннем слое}

Во внутреннем слое для синфазных колебательных состояний первого типа, определяемых (8) с учетом условий (12), поток имеет вид

$$
N_{0}=a\left|\psi_{0}\right|^{2}\left\{1-\operatorname{th}^{2}\left(q_{0} a\right)+\operatorname{th}\left(q_{0} a\right) / q_{0} a\right\} .
$$

Из (44) можно получить оценки потока в двух предельных случаях

$$
N_{0}= \begin{cases}a\left|\psi_{0}\right|^{2}\left\{2-\left(q_{0} a\right)^{2}\right\}, & q_{0} a \ll 1 \\ \left|\psi_{0}\right|^{2} / q_{0}, & q_{0} a \gg 1 .\end{cases}
$$

Если характеристики сред во внутреннем и внешних слоях одинаковы, то из (45) в основном приближении, используя (27), можно получить оценки потоков в виде

$$
N_{0}= \begin{cases}2 a\left(U_{2}-U_{1}\right) /\left(W_{1}-W_{2}\right), & q_{0} a \ll 1 \\ \left(U_{2}-U_{1}\right) / m\left(U_{1} W_{2}-U_{2} W_{1}\right), & q_{0} a \gg 1 .\end{cases}
$$

Во внутреннем слое для противофазных колебательных состояний первого типа, определяемых (8) с учетом условий (13), поток имеет вид

$$
N_{0}=a\left|\psi_{0}\right|^{2}\left\{1-\operatorname{cth}^{2}\left(q_{0} a\right)+\operatorname{cth}\left(q_{0} a\right) / q_{0} a\right\} .
$$

Из (42) можно получить оценки потока в двух предельных случаях

$$
N_{0}= \begin{cases}a\left|\psi_{0}\right|^{2}, & q_{0} a \ll 1 \\ \left|\psi_{0}\right|^{2} / q_{0}, & q_{0} a \gg 1 .\end{cases}
$$

Из (45) и (48) видно, что оценки потоков для синфазных и противофазных состояний первого типа в пределе $q_{0} a \gg 1$ одинаковы, как и следовало ожидать.

Если характеристики сред во внутреннем и внешних слоях одинаковы, то в пределе $q_{0} a \ll 1$ в основном приближении из (48), используя амплитуду (27), можно получить поток в виде

$$
N_{0}=\frac{a\left(U_{2}-U_{1}\right)}{\left(W_{1}-W_{2}\right)} .
$$

Величины потоков длинноволновых синфазных и противофазных колебательных состояний, как видно из (46) и (49), во внутреннем слое различаются в два раза.

Во внутреннем слое для синфазных колебательных состояний второго типа, определяемых (14) с учетом условий (18), поток имеет вид

$$
N_{0}=a\left|\psi_{0}\right|^{2}\left\{1+\operatorname{tg}^{2}(k a)+\operatorname{tg}(k a) / k a\right\} .
$$

В длинноволновом пределе $k a \ll 1$ из (50) получается оценка

$$
N_{0}=a\left|\psi_{0}\right|^{2}\left\{2+(k a)^{2}\right\} \text {. }
$$


Во внутреннем слое для противофазных колебательных состояний второго типа, определяемых (14) с учетом условий (19), поток имеет вид:

$$
N_{0}=a\left|\psi_{0}\right|^{2}\left\{1+\operatorname{ctg}^{2}(k a)+\operatorname{ctg}(k a) / k a\right\} .
$$

В длинноволновом пределе $k a \ll 1$ из (52) получается оценка, совпадающая с (48) при $q_{0} a \ll 1$.

Во всех рассмотренных случаях компоненты потоков конечны и отличны от нуля при условии, что параметры двух границ раздела слоев должны быть различными и обязательно обладать нелинейным откликом.

\section{7. Заключение}

В работе рассмотрены особенности локализации возбуждений в трехслойной структуре, в которой слои из линейных кристаллов разделены границами, характеризующимися собственным нелинейным откликом. В основе модели положено стационарное НУШ с потенциалом, моделирующим взаимодействие волны с границами раздела слоев в короткодействующем приближении. Нелинейность внутри границ раздела слоев считается керровского типа.

Основное внимание было уделено тому, что данная трехслойная структура является несимметричной в том смысле, что характеристики слоев и двух их границ раздела являются различными. Показано, что в такой несимметричной структуре могут существовать локализованные состояния двух типов. Такие состояния различаются распределением поля во внутреннем слое, а также диапазоном существования: состояния первого типа существуют в интервале $E<\min \left\{\Omega_{1}, \Omega_{2}, \Omega_{0}\right\}$, а второго - в $\Omega_{0}<E<\min \left\{\Omega_{1}, \Omega_{2}\right\}$.

Для каждого случая получены дисперсионные соотношения, определяющие зависимости энергии от параметров системы. Получены коэффициенты затухания поверхностных волн в явном виде. Указаны условия локализации поля в зависимости от характеристик слоев и границ их раздела. При анализе существования локализованных состояний основное внимание уделялось комбинации знаков параметров границ раздела, определяющих их притяжение/отталкивание и самофокусировку/дефокусировку. Найдены энергии таких локализованных состояний, которые не существуют в абсолютно симметричной структуре без взаимодействия волны с границами раздела слоев, причем обязательно наличие нелинейного отклика границ.

Показано, что в несимметричной структуре, также как и симметричной, могут существовать синфазные и противофазные колебательные локализованные состояния, характеризующиеся равными по модулю, но одинаковыми и противоположными знаками амплитуд поля на границах раздела слоев соответственно.

Показано, что наличие взаимодействия волны с границами раздела слоев приводит к тому, что в одномерной симметричной потенциальной яме с бесконечно высокими стенками с нелинейным откликом локализованное состояние существует не всегда. Для существования локальных длинноволновых противофазных колебательных состояний в несимметричной трехслойной структуре необходимо взаимодействие волны с границами раздела слоев.

Установлено, что нелинейный отклик границ раздела слоев приводит к тому, что линейные волны обладают характерными для нелинейных волн свойствами, а именно, зависимостью энергии от квадрата амплитуды волны.

Полученные дисперсионные соотношения применены для анализа влияния параметров сред и границы их раздела на поток, переносимый поверхностными волнами. Поток может трактоваться с точки зрения теории бозе-эйнштейновской конденсации как полное число частиц в системе. Его также можно рассматривать как условие нормировки. Тогда фиксированный поток может выступать в качестве управляющего параметра, через который можно выразить какие-либо другие характеристики локализованных состояний.

Полученные оценки потоков энергии, уносимых нелинейными поверхностными волнами, имеют значение для проектирования волноводных систем с заданными характеристиками, а также устройств управления и хранения данных на основе слоистых сред $[38,39]$. Результаты работы могут найти применение для совершенствования различных переключателей в волноводах и ограничителях мощности, способных пропускать световые импульсы выше или ниже требуемого значения потока энергии $[40,41]$.

\section{Список литературы}

[1] А.С. Давыдов. Солитоны в молекулярных системах. Наук. думка, Киев (1984). 288 с.

[2] А.М. Косевич, А.С. Ковалев. Введение в нелинейную физическую механику. Наук. думка, Киев (1989). 304 с.

[3] Ю.С. Кившарь, Г.П. Агравал. Оптические солитоны. От волоконных световодов до фотонных кристаллов. Физматлит, М. (2005). 648 с.

[4] R. Carretero-González, J. Cuevas-Maraver, D. Frantzeskakis, N. Karachalios, P. Kevrekidis, F. Palmero-Acebedo. Localized Excitations in Nonlinear Complex Systems. Springer Science \& Business Media (2013). 432 p.

[5] Y.V. Kartashov, B.A. Malomed, L. Torner. Reviews of Modern Physics 83, 247 (2011).

[6] В.И. Горенцвейг, Ю.С. Кившарь, А.М. Косевич, Е.С. Сыркин. ФНТ 16, 1472 (1990).

[7] И.В. Герасимчук, А.С. Ковалев. ФНТ 26, 799 (2000).

[8] Y.V. Bludov, D.A. Smirnova, Yu.S. Kivshar, N.M.R. Peres, M.I. Vasilevsky. Phys. Rev. B 89, 035406 (2014).

[9] А.В. Чаплик. Письма в ЖЭТФ 105, 565 (2017).

[10] Н.В. Высотина, Н.Н. Розанов, А.Н. Шацев. Оптика и спектроскопия 124, 82 (2018).

[11] А.И. Буздин, В.Н. Меньшов, В.В. Тугушев. ЖЭТФ 91, 2204 (1986).

[12] В.Н. Меньшов, В.В. Тугушев. ЖЭТФ 120, 899 (2001). 
[13] В.Н. Меньшов, В.В. Тугушев. ФТТ 44, 1650 (2002).

[14] С.Е. Савотченко. Конденсированные среды и межфазные границы 19, 567 (2017).

[15] С.Е. Савотченко. Вестн. Воронеж. гос. ун-та. Сер. физ. Мат. 1, 44 (2018).

[16] С.Е. Савотченко. ЖЭТФ 153, 339 (2018).

[17] A.A. Sukhorukov, Yu.S. Kivshar. Phys. Rev. Lett. 87, 083901 (2001).

[18] A.A. Sukhorukov, Yu.S. Kivshar. J. Opt. Soc. Am. B 19, 772 (2002).

[19] И.В. Герасимчук. Журн. нано- електрон. фіз. 4, 04024 (2012).

[20] I.V. Shadrivov, A.A. Sukhorukov, Yu.S. Kivshar, A.A. Zharov, A.D. Boardman, P. Egan. Phys. Rev. E 69, 016617-1 (2004).

[21] О.В. Коровай, П.И. Хаджи. ФТТ 52, 2277 (2010).

[22] Л.В. Федоров, К.Д. Ляхомская. Письма в ЖТФ 23, 36 (1997).

[23] Б.А. Усиевич, Д.Х. Нурлигареев, В.А. Сычугов, Л.И. Ивлева, П.А. Лыков, Н.В. Богодаев. Квантовая электрон. 40, 437 (2010).

[24] И.В. Алименков. Компьютерная оптика 37, 88 (2013).

[25] Д. Михалаке, Р.Г. Назмитдинов, В.К. Федянин. Физика элементарных частиц и атомного ядра 20, 198 (1989).

[26] О.В. Коровай, П.И. Хаджи. ФТТ 45, 364 (2003).

[27] О.В. Коровай, П.И. Хаджи. ФТТ 50, 1116 (2008).

[28] I.V. Gerasimchuk, P.K. Gorbach, P.P. Dovhopolyi. Ukr. J. Phys. 57, 678 (2012).

[29] И.В. Герасимчук. ЖЭТФ 121, 596 (2015).

[30] S.E. Savotchenko. Mod. Phys. Lett. B 32, 1850120 (2018).

[31] С.Е. Савотченко. Конденсированные среды и межфазные границы 20, 255 (2018).

[32] С.Е. Савотченко. Письма в ЖЭТФ 107, 481 (2018).

[33] С.Е. Савотченко. Письма в ЖЭТФ 108, 175 (2018).

[34] С.Е. Савотченко. ЖЭТФ 154, 517 (2018).

[35] S.E. Savotchenko. Solid State Commun. 283, (2018); DOI:10.1016/j.ssc.2018.08.002.

[36] С.Е. Савотченко. Нелинейный мир 3, 25 (2018).

[37] B. Luther-Davies, G.I. Stegeman. Materials for spatial solitons. In: Spatial Optical Solitons / Eds S. Trillo, W.E. Torruellas. Springer-Verlag, N.Y. (2001). P. 19-35.

[38] И.С. Паняев, Д.Г. Санников. Компьютерная оптика 41, 183 (2017).

[39] T. Strudley, R. Bruck, B. Mills, O.L. Muskens. Light: Sci. Appl. 3, e207 (2014)

[40] D. Zhang, Z. Li, W. Hu, B. Cheng. Appl. Phys. Lett. 67, 2431 (1995).

[41] Naim Ben Ali. Chin. J. Phys. 55, 2384 (2017).

Редактор Т.Н. Василевская 